\title{
LA COMPLEJIDAD EN EL MARCO DE UNA PROPUESTA PLURIPARADIGMÁTICA
}

\author{
THE COMPLEXITY IN THE MARK OF THE \\ PLURIPARADIGMATIC PROPOSAL
}

Rocío Pérez Mesa

Yaír Alexánder Porras Contreras*

\begin{abstract}
Resumen
El propósito del siguiente artículo es presentar una reflexión sobre la pluriparadigmaticidad como una alternativa de comprensión de las distintas concepciones de mundo que construyen los sujetos. En este sentido, se realiza una disertación acerca de la necesidad de una propuesta emergente que considera el pensamiento complejo como punto de referencia para la enseñanza de las ciencias.

Palabras clave: Complejidad, pluriparadigmaticidad, sistema, atractor, fractal, estructura disipativa.
\end{abstract}

\section{Abstract}

The aim of this article is to present a deep reflection about pluriparadigmaticity as a choice of understanding the conceptions of the world through the subjects construction. In this way, it is an analysis performance related to the complex thought, like a reference for science teaching.

Key words: Complexity, pluriparadigmaticity, sistem, atractor, fractal, dissipative structures.

*Universidad Pedagógica Nacional, rociopm28@yahoo.es, yairporras@yahoo.es 


\section{INTRODUCCIÓN}

El argumento central del presente artículo constituye un punto de reflexión en torno a la construcción, por parte de los sujetos, de una concepción de mundo más compleja, en la que se presenta la coexistencia de diversos paradigmas ${ }^{1}$, que funcionan como marcos interpretativos de la realidad. En este sentido, se pretende abrir un espacio de discusión en favor de una mirada sistémica de los acontecimientos, resaltando la importancia de la convivencia y el encuentro de múltiples posturas explicativas con las que se construyen diferentes visiones de mundo. Dicha construcción implica admitir la existencia de cierta sinergia paradigmática en la resolución de problemas, que se ratifica con la utilización de determinadas estrategias de actuación en un contexto de significación, explicitándose la dimensión discursiva que emerge de las interacciones entre los sujetos que conforman el grupo social.

Así mismo, se considera que los consensos generados en un grupo social pueden contribuir a la construcción de paradigmas plurales que trascienden el estatus epistemológico en el que se propugna la validez de los "paradigmas oficiales", orientándose ahora a la consideración de los puntos de convergencia de los otrora paradigmas rivales ("oficiales" y "no oficiales"), en un intento por conciliar en un mismo crisol posturas muy

De acuerdo con Kuhn (1992) un paradigma es "una constelación de logros -conceptos, valores, técnicas, etc- compartidos por una comunidad cientifica y usados por ésta para definir problemas y soluciones legitimos". diversas. Esta propuesta explicaría la resistencia al cambio de ciertas generalizaciones acríticas, enmarcadas en la llamada metodología de la superficialidad (Carrascosa y Gil, 1985) o la coexistencia tanto de hipótesis ad hoc, concepciones alternativas o hipótesis científicas que se auscultan en etapas tempranas de la formación académica y que se conservan casi inmutables en la formación universitaria o la práctica profesional (Gutiérrez, 1996; Rodrigo y Correa, 1998; Pozo y Gómez Crespo, 1998; Campanario y Moya, 1999).

\section{El surgimiento de la pluriparadigmaticidad}

Somos testigos de una gran bifurcación en todos los niveles del actuar humano. Como sujetos que pertenecemos a una sociedad, asumimos un papel protagónico en la comprensión del entramado complejo en el que se constituye la evolución de los sistemas. De hecho, actualmente reconocemos que el caos, generador de orden, brinda elementos teórico-prácticos para interpretar las incertidumbres, las inestabilidades y el azar, posibilitando asumir las crisis como una oportunidad para legitimar las diferentes propuestas explicativas que se entretejen en una red de significados con los otros, con el ambiente y con la capacidad de autoorganizarnos, autoreplicarnos y tomar decisiones.

Comprender estas propuestas novedosas permite consolidar también una nueva perspectiva de aprendizaje, con el propósito de transformar las prácticas pedagógicas y construir una realidad más 
justa y, ¿por qué no?, más solidaria con la vida. Así mismo, consideramos que la persistencia del paradigma tradicional se atribuye a su lucha permanente contra las incertidumbres, menguando la tensión entre la verdad y una realidad externa, creando espacios de claridad y tranquilidad en aquellos puntos donde el caos y la indeterminación se hacen explícitos.

En este sentido, Morin (1998) plantea una nueva perspectiva de la realidad en donde la complejidad se constituye como un entramado de relaciones que demuestran la complementariedad de los eventos. De esta manera, dicha complementariedad viene a explicitarse con la analogía del holograma, al constituirse la parte como totalidad y ésta última ser mucho más que la suma de las partes, utilizando la sinergia propia de la interacción entre los sistemas.

Quizás estos principios constituyan la semilla de la crisis del paradigma tradicional, que en la ciencia y en la educación se ha caracterizado por la búsqueda constante, bajo el ideal racionalista, de la seguridad en el dominio de conceptos, leyes y teorías verdaderas. De hecho, una de las premisas del científico racional se centra en la institucionalización del conocimiento, creando comunidades cerradas que utilizan la objetividad como argumento para obligar a pensar (Maturana, 1997), al igual que crean una serie de criterios de demarcación entre las ciencias sociales y las ciencias experimentales, con lo que se procura aislar los fenómenos a pesar de las interacciones que pueden presentar entre sí.
Como vemos, la crisis del paradigma tradicional supone una nueva concepción de cultura, individuo, realidad, progreso y cambio, pero frente a la complejidad y a la teoría del caos, las perspectivas teórico-prácticas son aún limitadas. De hecho, muchos autores y colegas insisten en precisar el carácter de estas propuestas como un paradigma, mientras que otros las consideran un metalenguaje $\mathrm{o}$ una metodología. En palabras de Maldonado (1999), es posible identificar tres caminos hacia la lógica de la complejidad: la complejidad como método, la complejidad como cosmovisión y la complejidad como ciencia. Pretenderíamos entonces generar una reflexión en relación con el conocimiento que estos saberes permiten construir, siendo conscientes de las interacciones entre los diversos elementos que componen la dinámica propia de los sistemas, admitiendo lo importante de trascender el pensamiento hacia una actitud reconstructiva en torno a la vida, la naturaleza y el conocimiento.

Ejemplos muy puntuales de este trasegar entre el discurso novedoso y la práctica incipiente son aquellos planteamientos que promulgan las miradas amplias, pero intentan atomizar la realidad, almacenando así conocimientos propios de cada retícula. Sabemos que lo propio de lo vivo es la vida, pero desconocemos su sentido; intentamos desintegrar los sistemas hasta su última esencia, diseccionando subsistemas de órganos, tejidos y células. Así mismo, deseamos saber la constitución final de los sistemas fisicoquímicos y separamos de su contexto la 
partícula fundamental, ya sea un átomo, un nucleón, un quark. Hablamos de un cambio cultural, pero nos fijamos en los estáticos procesos sociales que globalizan o menosprecian la diversidad cultural, centrando su mirada en el "atesorar para sobrevivir", como leitmotiv del progreso.

En contraposición con esta visión de los acontecimientos, se cree que este es un período de dudas, indeterminaciones y problemas, que merecen ser examinados como parte de un mundo cambiante, en el que cobra sentido una nueva concepción de los fenómenos biológicos y culturales. En consecuencia, de acuerdo con las investigaciones contemporáneas, un punto de convergencia en el que se construye un nuevo marco unificado de explicación de los procesos biológicos, sociales y culturales es sin duda alguna la vida y, junto con ella, el entendimiento de la estructura y la organización de los sistemas. Resulta clave considerar que estos modelos explicativos son complementarios entre sí y constituyen uno de los múltiples marcos de referencia que ejemplifican la convivencia de diversos paradigmas.

En este sentido, se cree firmemente en la posibilidad de coexistencia de una multiplicidad de paradigmas, que a su vez potencian el surgimiento de una trama de interacciones entre sus estatutos epistemológicos y las metodologías emergentes que los caracterizan. En la aceptación de diversas concepciones de mundo se construye la nueva realidad y se establece la posibilidad de crear nue- vos mundos (Bruner, 1998), con base en la comprensión pluriparadigmática de los eventos.

La pluriparadigmaticidad consiste, entonces, en el establecimiento de una red de significación por parte de los sujetos, en un espacio relacional en el que las interacciones entre la pluralidad de paradigmas explicativos generados desde perspectivas diversas y complementarias funcionan como marcos de interpretación de la realidad. Este proceso de construcción personal de una concepción de mundo se consolida en la intersección de diferentes dominios ${ }^{2}$ de interacciones que caracterizan cada paradigma; tal fenómeno se corresponde con una progresión desde un pensamiento simple a uno complejo, mediada por la articulación entre las posiciones de los individuos, sus micromundos y las ofertas simbólicas del colectivo, enmarcadas a su vez en el lenguaje.

\section{La pluriparadigmaticidad: una perspectiva para la enseñanza de las ciencias}

Esta propuesta pretende aportar elementos de discusión en torno a la enseñanza de las ciencias desde una perspectiva compleja, debido a la cada vez más cambiante red de fenómenos que se vislumbran desde diferentes ámbitos del conocimiento. De hecho, somos testigos de la incursión de una serie de modelos que explican los fenómenos, entre ellos la

En esta propuesta consideramos los dominios explicativos como un sistema de conocimientos que se construyen en un contexto particular de significación y que están determinados por el criterio de validación de las afirmaciones que les son propias, especificando un modo de actuar en él. 
complejidad, la teoría del caos, la teoría de sistemas, etc., que en el ámbito educativo son motivo de discusión, mostrando un amplio espectro de relaciones que se pueden establecer a nivel conceptual, metodológico, actitudinal, afectivo, axiológico y deontológico.

\section{La pluriparadigmaticidad: integrando lo social y lo cultural}

Las anteriores evidencias son sólo algunos de los muchos ejemplos que existen en la naturaleza y permiten analizar, desde la diversidad del pensamiento, una propuesta que concilia la dialogicidad, la dialéctica, los consensos y los disensos, frente a los muchos paradigmas explicativos que la mente construye. De hecho, nuestra misma concepción de cultura no escapa de estas miradas, que de por sí sobrepasan las posiciones simples, que enajenan y discriminan. Sólo una comprensión borrosa de la cultura nos permite abordar los imaginarios colectivos como una red de significados, "plagados de elipsis, de incoherencias, de sospechosas enmiendas y de comentarios tendenciosos, y además escrito no en las grafías convencionales de representación sonora, sino en ejemplos volátiles de conducta modelada" (Geertz, citado por Rey, 2003).

Aquí se presenta la cultura como una manifestación de la sociedad que explícita sus imaginarios, sus representaciones y que merece una caracterización compleja a partir de las interacciones que sub-

3 "No se puede meter la totalidad en el bolsillo, porque el bolsillo es parte de la totalidad, por lo tanto hay un agujero en él" (Briggs; Peat, 1999). yacen en su seno. Así mismo, como en la analogía del agujero ${ }^{3}$, la cultura debe ser vista como un todo que surge de la riqueza y diversidad de interacciones entre sus partes, de tal manera que para su estudio y análisis es prudente seguir las palabras de Geertz (citado por Rey, 2003), quien propone que "la cultura ha de ser no una ciencia experimental en busca de leyes, sino una ciencia interpretativa en búsqueda de significaciones".

Consecuentemente, la pluriparadigmaticidad permite vislumbrar la articulación de lo social, lo cultural y las redes biológicas, que, en palabras de Capra (2003), son "un patrón no lineal de organización, por lo que es muy probable que las ideas y los conceptos desarrollados en la teoría de la complejidad, como la retroalimentación y la emergencia, sean también relevantes en el contexto social". Dichas redes sociales constituyen una trama de la que la comunicación es su nodo integrador, por lo que es válido afirmar que existimos en el lenguaje.

En este orden de ideas, surge la posibilidad de intervenir y procurar un cambio social a partir de una mirada sistémica y pluriparadigmática, que bajo un esfuerzo interpretativo cuestione el conocimiento amparado en los saberes rigurosos y las ciencias clásicas, puntos oscuros en donde la fragmentación y el uso de un método científico proveen de seguridad al indagar los secretos que la naturaleza esconde. "Se trata entonces de comprender que desde hace cierto tiempo el conocimiento ha dejado de ser dominio exclusivo de los intelectuales y sus herederos (investiga- 
dores, "ingenieros sociales" o analistas simbólicos) y se ha convertido en un medio común y en un importante dispositivo mediante el cual las sociedades se organizan, cambian y se adaptan a las nuevas circunstancias históricas" (Cubides y Durán, 2002).

Así, sería conveniente seguir cuestionándonos en relación con el cambio social y las transformaciones revolucionarias que se vienen dando en todos los ámbitos del conocimiento. De hecho, la evolución de las representaciones no sigue un tratamiento lineal, y podríamos decir que presenta bifurcaciones e indeterminaciones, puntos en los que se generan diversos paradigmas filosóficos, científicos, sociales y culturales que conviven "simbióticamente". Consecuentemente, es prudente pensar que los paradigmas exhiben discontinuidades, en ciertos casos sutiles, que son la semilla de la evolución de los sistemas. La resistencia a nuevas formas de pensamiento y la imposibilidad de crear nuevos mundos, quizás es uno de los retos más interesantes en el reconocimiento de la complejidad y el caos en la cultura.

\section{La educación desde una mirada plural}

Manifestaciones coincidentes con estas propuestas suponen que la educación y en particular la pedagogía, entendida como un saber reconstructivo, deben trascender hacia formas más globales de interpretación de los procesos de construcción de los imaginarios colectivos (Porlan, 1990), haciendo también evidente la ruptura de modelos geométricos mentales y la apertura de los compartimentos disciplinares, privilegiados antaño por visiones estáticas que plagan de prejuicios a los colectivos humanos. Estas posiciones parcializadas favorecen múltiples formas de intimidación debido a que sobreponen el interés de la parte a las necesidades del colectivo, en una clara defensa de posiciones tendenciosas que niegan la posibilidad de construir realidades.

Compartimos con Vattimo, la necesidad de configurar una hermenéutica pluralista que consolide la multiplicidad de interpretaciones sobre los fenómenos y cuestione los alcances de una realidad objetiva. Por tal razón, se acepta que la hermenéutica es la "filosofía que pone en su centro el fenómeno de la interpretación; es decir, un conocimiento de lo real que no se piensa como reflejo objetivo de las cosas 'allá afuera', sino como una comprensión que lleva en sí el sello de quien conoce" (Vattimo, 1998).

A nivel hermenéutico, el estudio de los sistemas sociales desde la complejidad nos ha enseñado que mientras más examinamos las propiedades caóticas de los sistemas, más conocemos de nosotros mismos.

De hecho, valdría la pena dirigir la mirada a los procesos culturales que insisten en institucionalizar las sociedades bajo una finalidad amparada por un paradigma sombrío, que en últimas se traduce en dominación y ocupación. Dentro de estos procesos culturales, la educación ha movilizado otros caminos para 
contrarrestar aquella doctrina compartimentada, al reconocer el pensamiento relacional, la diversidad, lo novedoso, los atractores y las bifurcaciones.

\section{El aprendizaje como un proceso multidimensional}

De acuerdo con los planteamientos educativos expresados hasta aquí, resulta procedente considerar un paradigma como un marco de compromisos centrales que organizan el aprendizaje de los estudiantes, por lo que aquel cambio conceptual que propugna el reemplazo de las viejas concepciones por otras novedosas queda restringido, al desconocer la posibilidad que tienen los estudiantes de emplear concepciones pluriparadigmáticas, aplicables cada una de ellas a contextos particulares de significación (micromundos) ${ }^{4}$.

Así, la enseñanza de las ciencias promueve, desde una perspectiva compleja, la reconstrucción de los procesos culturales de comunicación, en lo que al fortalecimiento de la cultura escolar se refiere, asumiéndose la dialéctica y el diálogo entre lógicas plurales (dialogicidad), como una forma de interactuar y dar sentido a la realidad. De esta manera, el objetivo de la enseñanza de las ciencias de procurar un cambio conceptual se replantea para abordar también aspectos metodológicos, actitudinales, afectivos, éticos y sociológicos.

\footnotetext{
${ }^{4}$ Un micromundo, según Varela (1996), se define como "un espacio donde un ser humano se constituye como tal en un sistema social donde hay una recurrencia en una interacción de cooperación y se establece una autoconciencia”.
}

De acuerdo con lo anterior, sin un enfoque metodológico las ideas de los estudiantes se encuentran descontextualizadas, pues un exclusivo cambio conceptual podría asociarse con un simple cambio del contenido de las concepciones, recalcándose una vez más los conocimientos declarativos en contra del aprendizaje significativo. Por esta razón se hace prioritario aproximar los estudiantes a las características del trabajo científico, como la interpretación de situaciones problema, la emisión de hipótesis, el diseño experimental, el análisis de resultados, su coherencia global y demás actividades propias de un proceso de aprendizaje de las ciencias, como investigación orientada.

Gil y sus colaboradores (1993) proponen unas estrategias flexibles de trabajo enmarcadas en el aprendizaje de las ciencias como investigación orientada (Ramírez Castro, 1990; Gil, 1999):

1. Se plantean situaciones problemáticas que motiven al estudiante y que proporcionan una concepción preliminar de la tarea.

2. Se propone un estudio cualitativo de las situaciones problemáticas y la toma de decisiones, utilizando búsquedas bibliográficas, con el fin de que los estudiantes delimiten el problema y expliciten sus ideas.

3. Los problemas se trabajan siguiendo una orientación científica con el objeto de que se emitan hipótesis (explicitándose las ideas previas), se elaboren estrategias de resolución y se cotejen los resultados con los de otros grupos; 
espacio propicio este para los conflictos cognoscitivos y el análisis de nuevas hipótesis.

4. Los nuevos conocimientos se aplican a nuevas situaciones para profundizar y afianzarlos, haciéndose énfasis en las relaciones ciencia, tecnología, sociedad y ambiente. En todo el proceso es prioritario favorecer las actividades de síntesis, la elaboración de productos como mapas conceptuales, memorias, etc., y la concepción de nuevos problemas.

Como vemos, en el caso de la enseñanza de las ciencias, el objetivo del aprendizaje sería explicitar las teorías personales y la transformación progresiva hacia posiciones próximas a paradigmas explicativos. Bajo esta perspectiva, los paradigmas dejan de ser marcos conceptuales aislados desde donde se descartan o se aprueban cierto tipo de intervenciones de un determinado grupo social y se convierten en un marco de compromisos centrales que aportan desde su propio estatuto epistemológi$\mathrm{co}$, tanto conceptos nodales, atractores, problemas y/o metodologías de trabajo, favoreciendo de esta manera la integración. Ya no se hablaría de disciplinas herméticas, sino de paradigmas comunes, que ven en las disciplinas el camino y no la génesis en la comprensión de los sistemas de una realidad global.

En este sentido, la propuesta pluriparadigmática centrada en la complejización de los procesos de enseñanza y aprendizaje de las ciencias retoma la visión integradora del pensamiento sisté- mico, al concebir la diversidad, la reciprocidad, las interacciones y la cooperación como elementos comunes a las diferentes propuestas de interpretación y transformación de la realidad.

\section{Los micromundos y los procesos de aprendizaje}

La tarea que surge después de esta exposición consiste en establecer cuáles son esos micromundos y microidentidades que emergen en nuestra convivencia con el otro ${ }^{5}$. De hecho, los diferentes dominios explicativos (la física, la química, la biología, lo ambiental, el arte, etc.) posibilitan el establecimiento de múltiples micromundos y microidentidades. En consecuencia, el mundo se construye constantemente en comunión con los otros; la educación como proceso en el que se reconstruye el lenguaje permite enfocar la labor del docente hacia la dinamización de una cultura, construida a partir de las transiciones recurrentes entre micromundos, fundamento por el cual se consolida una propuesta emergente.

Estos planteamientos se pueden ejemplificar de distintas formas. Tal es el caso de la conceptualización de la energía, término particularmente complejo, que en la historia de la ciencia ha evolucionado desde planteamientos que la catalogan como una sustancia cuasimaterial; un fluido; cierta capacidad para realizar un trabajo; aquella capacidad para producir cambios o una magnitud física fundamental de los sistemas en razón de la cual pueden mo-

${ }^{5}$ Varela (1996) denomina microidentidades a ciertas disposiciones para la acción que circulan en correspondencia con determinados micromundos. 
dificar su estado, así que se transforma, se transmite y se degrada, dependiendo del sistema de referencia y, fijado este, se conserva. De acuerdo con estos referentes teóricos, algunas investigaciones muestran que pueden coexistir diferentes concepciones de energía en un mismo individuo, resaltándose aquellas que la consideran como una sustancia cuasimaterial, un fluido y una capacidad para realizar un trabajo (Porras, 2005). Confirmamos de esta manera la presencia de un lenguaje tradicional, pero limitado, que privilegia aspectos relacionados con la fuerza o el movimiento, trasladándose de nuevo la idea de "vis viva", que surge, para el caso de choques perfectamente elásticos, de la conservación tanto de la cantidad de movimiento, como de la "fuerza viva" (el producto de la masa por la velocidad al cuadrado), origen histórico del principio de conservación de la energía.

Como vemos, el aprendizaje del concepto energía requiere aunar una serie de elementos paradigmáticos que permitan trascender la visión sesgada con la que los estudiantes interpretan su significado y sentido. Así, resulta particularmente difícil interpretar el hecho de que todos los sistemas tienen energía, pero no todos realizan trabajo, proponiéndose para ratificar esta afirmación que existen sistemas que sólo tienen energía interna y no producen transformaciones, debido a que se encuentran a la misma temperatura del ambiente. En este sentido, la confusión, generalizada tanto en los libros de texto como en el lenguaje cotidiano, de considerar el trabajo y el calor como formas de energía ratifica la limitación en el plano teórico-práctico de asociar estos conceptos con procesos de intercambio de energía.

Otro ejemplo que muestra una visión pluriparadigmática en la enseñanza de las ciencias lo constituye la indagación de las concepciones de Ambiente, siguiendo una metodología que va desde la formulación de una pregunta, la elaboración de un dibujo y el diseño de un mapa conceptual. Obteniendo como resultado diferentes formas de concebirlo, de acuerdo con cada instrumento de evaluación.

En relación con la pregunta ¿Qué es el ambiente?, los estudiantes lo conciben, en su mayoría, como el conjunto de factores bióticos y abióticos y, en una menor proporción, como las relaciones que se establecen entre lo biofísico y el hombre. Al dibujarlo, aumenta el porcentaje de estudiantes que lo representa como un recurso para aprovechar y satisfacer las necesidades del hombre. Otro grupo muestra el ambiente como un espacio para conocer sus dinámicas, sus interrelaciones y la diversidad que puede presentar, lo cual amplia el panorama en relación con el primer escrito (Pérez, 2004).

Finalmente, al elaborar un mapa conceptual explicitan la idea de ambiente como un conjunto de factores bióticos y abióticos que se interrelacionan, lo cual muestra un avance hacia una perspectiva que establece relaciones entre lo biofísico; sin embargo, otro grupo de estudiantes incluyen al ser humano como parte integrante del ambiente, lo cual se aproxima a una concepción más 
compleja. Como vemos, un grupo considerable de estudiantes se desempeña de modo diferente en cada uno de los contextos de indagación que hacen parte del proceso de construcción de conocimiento, mientras que para otro grupo se mantiene la concepción inicial de ambiente.

De acuerdo con lo anterior, podemos considerar que nuestra vida está constituida por micromundos relativamente estables, en los que desplegamos nuestras identidades, nuestras formas de ver y de concebir el mundo. Reconocemos que existen situaciones en las que nuestra mera presencia en distintos dominios no está regida por las mismas regularidades de otros micromundos. Sería necesario reconocer el significado y el sentido que manifiestan dichos micromundos, a partir de las interacciones que se entretejen en un contexto relacional, cuando consideramos que existimos en el lenguaje y construimos una amalgama de dominios explicativos. Estos últimos constituyen un entramado de experiencias en las cuales se reformulan las explicaciones que hacen las personas, bien sea frente a una pregunta o un problema y, con base en experiencias distintas, legitiman determinadas respuestas. Así, el explicar consiste en generar juicios de valor que en sí mismos reformulan experiencias, hecho por el cual se va construyendo paulatinamente un marco explicativo con que se siente identificado el sujeto. Vale la pena señalar que este trasegar entre diferentes micromundos puede generar rupturas de los paradigmas explicativos del sujeto, que procuran la emergencia de respues- tas y acciones que permiten conciliar la necesidad de pensar en otras visiones de mundo, al enriquecer los aprendizajes obtenidos en un micromundo.

De acuerdo con el dominio explicativo propio del micromundo en el que se genera un problema, la elaboración de una respuesta aceptable, que dé cuenta de los intereses, expectativas y conocimientos que emergen del lenguaje, viene a ser evaluada con base en los criterios de validación de las explicaciones, que son establecidos por las conductas relacionales de los colectivos, considerándose la autonomía como un proceso de extensión de redes que ponen en comunicación diferentes micromundos (Pérez, et al, 2004). Es prioritario pensar que cada micromundo que se construye es una oportunidad para crear criterios de validación, cuya génesis, constituida en un acto consciente de los individuos, es la ocasión perfecta para cimentar una concepción más solidaria con la vida. Muy probablemente uno de los múltiples factores por los cuales es considerada la ciencia con un estatus superior, frente a otros dominios explicativos, que no deben considerarse superficiales con respecto a esta, se centra en el esfuerzo riguroso que las comunidades académicas despliegan al elaborar determinados criterios de validación de sus explicaciones científicas.

En la enseñanza de las ciencias resulta particularmente enriquecedor generar espacios de discusión en torno a la diversidad de discursos que utilizan los estudiantes para explicar y modificar 
los significados que construyen en su relación con los otros, resaltando el papel de las interacciones discursivas que emergen de la propuesta de aprendizaje por investigación. Por esta razón se cree firmemente en la posibilidad de coexistencia de una multiplicidad de paradigmas que potencializan el surgimiento de una trama de interacciones. Un aspecto fundamental dentro de la propuesta pluriparadigmática consiste en establecer no sólo la complejidad en la interacción de los conceptos propios del dominio explicativo de las ciencias, sino en las diferentes concepciones que subyacen a los micromundos en los que el sujeto se desenvuelve. Así, algunos aspectos a considerar dentro de dicha propuesta suponen el desarrollo de una hipótesis de progresión que consolide la conexión de mundos, a partir del desarrollo de un pensamiento sistémico para la resolución de problemas y la toma de decisiones. Entre estos aspectos se encuentran:

- La legitimación de un espacio relacional. Establecimiento de interacciones multinivel a través del tratamiento problémico.

- Reconocimiento de los paradigmas explicativos de los sujetos (ideas alternativas, hipótesis ad-hoc o concepciones científicas)

- Encuentro de los diferentes dominios de interacciones que caracterizan cada paradigma (consensos y disensos).

- Articulación entre las concepciones del sujeto y las ofertas simbólicas del colectivo (pares cognitivos), a la luz de los paradigmas explicativos diversos (docente-aula de clase-comunidad académica).

- Reconstrucción de los procesos de comunicación involucrados en el desarrollo de la cultura escolar. Interacción entre los dominios relacionales (emociones) y las coordinaciones consensuales de conducta (lenguaje).

- Validación y explicitación del contexto de interlocución (viabilidad de las hipótesis, contrastación de las formas de resolución de problemas, pertinencia de los diseños, etc.).

- Establecimiento de una red de significación enriquecida por la pluralidad de paradigmas explicativos (pluriparadigmaticidad).

- Desarrollo de una hipótesis de progresión que evidencie una transición hacia formas complejas de explicación (Necesidad de un pensamiento sistémico a través de la sinergia de los actos de los individuos).

- Reconocimiento de la pluriparadigmaticidad como punto de encuentro de explicaciones diversas.

- Formulación de nuevos problemas. Elaboración de productos, síntesis, esquemas, mapas conceptuales, etc. (Evaluación de los aprendizajes y los modelos mentales de los sujetos).

- Retroalimentación permanente del proceso de aprendizaje.

Lo anterior nos plantea un reto que exige no sólo cambios conceptuales y epistemológicos, sino también transformaciones en las metodologías que em- 
pleamos y enseñamos a utilizar a nuestros estudiantes; trascender hacia formas más complejas de comprender el mundo supone el establecimiento, bajo consen-

\section{Bibliografía}

Briggs, J., y Peat, F. (1999). Las siete leyes del caos. las ventajas de una vida caótica. Grijalbo.

Bruner, J. (1998). Realidad mental y mundos posibles. Barcelona: Gedisa.

Campanario, J., y Moya, A. (1999). ¿Cómo Enseñar Ciencias?, Principales Tendencias y Propuestas. En Enseñanza de las ciencias. 17 (2), pp. 179-192.

Carrascosa, A., y Gil, D. (1985). "Metodología de la Superficialitat" il'aprenentatge de les Ciéncies. En Enseñanza de las Ciencias. pp. 113-120.

Capra, F. (2003). Las conexiones ocultas. Implicaciones sociales, medioambientales, económicas y biológicas de una nueva visión del mundo. Editorial Anagrama.

Cubides, H., y Durán, A. (2002). Epistemología, ética y política en la relación entre investigación y transformación social. En Nómadas. No. 17. Bogotá: Universidad Central.

Gil, D. (1993). Contribución de la historia y de la filosofía de las ciencias al desarrollo de un modelo de enseñanza/aprendizaje por investigación. En Enseñanza de las ciencias 11 (2). pp. 197-212.

Gil, D. (1999). El modelo constructivista de enseñanza/aprendizaje de las ciencias: una corriente innovadora fundamentada en la investigación. Organización de Estados Iberoamericanos. www.oei.org.co/oeivirt/gilo2.htm.

Gutiérrez, R. (1996). Modelos mentales y concepciones espontáneas. En Alambique. No. 7. Enero. pp 73-86. so, de diversas alternativas de interpretación, que involucran interpretaciones múltiples, flexibles, divergentes y pluriparadigmáticas, de complejidad creciente.

Kuhn, T. S. (1992). La estructura de las revoluciones científicas. México: Fondo de Cultura Económica.

Maturana, H. (1997). La Objetividad. Un argumento para obligar. Santiago: Dolmen.

Maldonado, C. E. (1999). Esbozo de una filosofía de la lógica de la complejidad. En Visiones sobre la Complejidad. Bogotá: Ediciones El Bosque.

Morin, E. (1998). Introducción al pensamiento complejo. Barcelona: Gedisa.

Pérez, et al. (2004). Las condiciones de autonomía en los estudiantes del proyecto curricular en Li cenciatura en Biología. Informe Final. Universidad Pedagógica Nacional. Bogotá, Colombia. Proyecto de Investigación.

Pérez, (2004). La construcción de la categoría ecorregión en estudiantes de IV semestre de la Licenciatura en Ciencias Sociales de la Universidad Pedagógica Nacional. Maestría en Educación con énfasis en Educación Ambiental. Universidad Pedagógica Nacional. Bogotá, Colombia. Tesis de grado.

Porlán, R. (1990). Hacia una fundamentación epistemológica de la enseñanza. En Investigación en la Escuela. No. 10. pp 3-21.

Porras, Y. (2005). El aprendizaje significativo de los conceptos calor, temperatura y energía ilnterna: Una concreción del modelo de aprendizaje de la química como investigación orientada. Maestría en Docencia de la Química. Universidad Pedagógica Nacional. Bogotá, Colombia. Tesis de grado. 
Pozo, J. I., y Gómez Crespo, M. A. (1998). Aprender y enseñar ciencias. Del conocimiento cotidiano al conocimiento científico. Madrid: Morata.

Ramírez Castro, J. L. (1990). La Resolución de Problemas de Física y de Química como Investigación en la Enseñanza Media: Un Instrumento de Cambio Metodológico. Universidad Autónoma de Barcelona. Tesis de Doctorado.

Rey, G. (2003). Contrapanfleto a los estragos del mal de ojo. En El Malpensante. Bogotá. Enero.
Rodrigo, M. J., y Correa, N. (2000). Teorías implícitas, modelos mentales y cambio educativo. En J. I. Pozo \& C. Monereo (Eds.), EI aprendizaje estratégico (pp. 75-86). Madrid: Santillana, Aula XXI.

Varela, F. (1996). Ética y acción. Santiago de Chile: Dolmen Ediciones

Vattimo, G. 2002. La posmodernidad a debate. Simposio Internacional "La Posmodernidad a debate" (1998, Bogotá). Universidad Santo Tomás. Biblioteca Colombiana de Filosofía.

ARTíCULO RECIBIDO: 24-1 1-2004

Y APROBADO: 17-06-2005 